Pacific Journal of Mathematic 


\section{CLOSED SYSTEMS OF FUNCTIONS AND PREDICATES}

\section{DAVID GEIGER}

In this paper we show that there is a one to one correspondence between systems of functions defined on a finite set $A$ and systems of predicates defined on $A$. This result implies that a complete set of invariants for a universal algebra on $A$ is given by predicates defined on $A$. Conversely functions on $A$ provide a complete system of invariants for sets of predicates closed under conjunction, change of variable and application of the existential quantifier.

We begin in $\S 2$ by giving a definition of closure for systems of functions and predicates. This is followed by a definition of commutivity of a function and a predicate which gives a correspondence between the two types of systems. In Theorems 1 and 2 of $\S 3$ we show that the correspondence is a Galois connection. In Theorem 3 we consider sets of predicates closed under the existential quantifier and show that the corresponding systems are determined by functions defined for all values of the arguments. In Theorems 4 and 5 we include disjunction and then negation in the definition of closure of a set of predicates. We also require that equality be among the predicates. The corresponding systems consist of essentially first order functions and essentially first order permutations respectively. We conclude in $\S 4$ with some comments on the infinite case and some general comments on these results.

2. Basic definitions. Associated with any subset of $A^{n+1}$, the set of all sequences of length $n+1$ with elements in $A$, is the $n$-th order function $f\left(x_{1}, \cdots, x_{n}\right)$ which may be many valued and may not be defined on all of $A^{n}$. A system of functions $\mathscr{P}$ is defined to be closed if the following conditions are satisfied:

(i) $\mathscr{L}$ is closed under composition.

(ii) If $f\left(x_{1}, \cdots, x_{n}\right) \in \mathscr{L}$ is associated with the subset $P \subset A^{n+1}$ then any $g\left(x_{1}, \cdots, x_{n}\right)$ associated with $Q \subset P$ is in $\mathscr{L}$.

(iii) For any $n, \mathscr{L}$ contains all functions $f$ defined on $A^{n}$ such that $f\left(x_{1}, \cdots, x_{n}\right)=x_{i}$.

In defining closed systems of predicates the author has the following model in mind. We are given a sequence $A_{1}, A_{2}, A_{3}, \cdots$ of sets of predicates, each $A_{i}$ containing all subsets of $A^{i}$. For each $A_{i}$ a set of operators isomorphic to $\mathscr{S}_{i}$ the symmetric group is given which maps $A_{i}$ onto $A_{i}$. These correspond to permutations of the variables 
of predicates in $A_{i}$. There is an operator $R: A_{i+1} \rightarrow A_{i}$ which takes $P\left(\left(x_{1}, \cdots, x_{i+1}\right)\right.$ to $P\left(x_{1}, x_{1}, x_{2}, \cdots, x_{i}\right)$ and an operator $E: A_{i+1} \rightarrow A_{i}$ which takes $P\left(x_{1}, \cdots, x_{i+1}\right)$ to $(\exists y) P\left(y, x_{1}, \cdots, x_{i}\right)$. Also there is an operator $A: A_{i} \rightarrow A_{i+1}$ which corresponds to the cartesian product with $A$ or to the introduction of a dummy variable. Thus $\left(x_{1}, \cdots, x_{i+1}\right) \in A P$ if and only if $\left(x_{2}, \cdots, x_{i+1}\right) \in P$. A predicate in $A_{i}$ will be said to have order $i$. A system $\mathscr{P}$ of predicates is defined to be closed if it satisfies the following conditions:

(i) If $P \in \mathscr{P}$ and $Q \in \mathscr{P}$ and $P$ and $Q$ have the same order then $P \cap Q \in \mathscr{P}$.

(ii) If $P \in \mathscr{P}$ then any predicate obtained from $P$ by permuting the variables is in $\mathscr{P}$.

(iii) If $P \in \mathscr{P}$ then $A P$ and $R P$ are contained in $\mathscr{P}$.

(iv) $\mathscr{P}$ contains the first order predicate $A$.

Now we define commutivity of a function and a predicate. Let $M$ be an $n \times m$ matrix with elements in $A$, then we write $M \subset P$ where $P$ is an $m$-th order predicate if each row of $M$ is a sequence contained in $P$. If $N$ is an $m \times n$ matrix and $f$ is an $n$-th order function then $f(N)$ is the $m \times 1$ column matrix obtained by letting $f$ operate on each row of $N$. If $f$ is not defined for some row of $N$ we say that $f(N)$ is not defined. The predicate $P$ commutes with the function $f$ if for every $M \subset P$ the row matrix $f\left(M^{T}\right)^{T}$ when defined is a sequence contained in $P$. Here $M^{T}$ is the transpose matrix of $M$. If $\mathscr{L}$ and $\mathscr{P}$ are systems of functions and predicates we write $\mathscr{L}^{*}$ and $\mathscr{P}^{*}$ for the systems of predicates and functions respectively which commute with $\mathscr{L}$ and $\mathscr{P}$.

3. Main results. It can be verified that $\mathscr{L}^{*}$ and $\mathscr{P}^{*}$ are closed systems. We will show that if $\mathscr{L}$ and $\mathscr{P}$ are closed systems then $\mathscr{L}=\mathscr{L}^{* *}$ and $\mathscr{P}=\mathscr{P}^{* *}$.

THEOREM 1. If $\mathscr{L}$ is a closed system of functions then $\mathscr{L}=$ $\mathscr{L}^{* *}$.

Since $\mathscr{L} \subset \mathscr{L}^{* *}$ we need only show that for any function $g\left(x_{1}, \cdots, x_{m}\right)$ not in $\mathscr{L}$ there exists a predicate in $\mathscr{L}^{*}$ which does not commute with $g$. Assume that $g$ is defined only on the sequences $s_{1}, s_{2}, \cdots, s_{k}$. We form the $k \times m$ matrix $T$ with $i$-th row equal to $s_{i}$. For any function $f\left(x_{1}, \cdots, x_{r}\right)$ in $\mathscr{L}$ and any $k \times r$ matrix $F$ with columns taken from $T$ we form the column matrix $f(F)$. If $f(F)$ is not a column of $T$ we adjoin it to $T$ and get a $k \times(m+1)$ matrix $T_{1}$. In this way we can adjoin columns to $T$ until we finally reach a matrix $T_{0}$ with $k$ rows such that for any function $f$ in $\mathscr{L}$ and any matrix $F$ with columns from $T_{0}$ the column matrix $f(F)$ 
will be in $T_{0}$ if it is defined. If $g(T)$ is a column of $T_{0}$ then $g$ can be derived from functions in $\mathscr{L}$ so we can assume that $g(T)$ is not in $T_{0}$. From $T_{0}$ we form the $k$-th order predicate $P_{0}$ which contains all the rows of $T_{0}^{T}$. It is evident that $P_{0}$ is in $\mathscr{L}^{*}$ but does not commute with $g$. Thus $\mathscr{L}=\mathscr{L}^{* *}$.

THEOREM 2. If $\mathscr{P}$ is a closed system of predicates then $\mathscr{P}=$ $\mathscr{P}^{* *}$.

Since $\mathscr{P} \subset \mathscr{P}^{* *}$ we need only show that for any $n$-th order

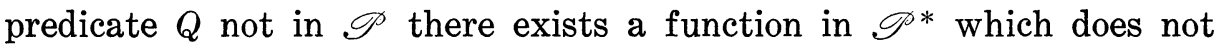
commute with $Q$. Let $P$ be the intersection of all $n$-th order predicates of $\mathscr{P}$ which contain $Q$. Let $s_{1}, s_{2}, \cdots, s_{k}$ be all the $1 \times n$ matrices contained in $Q$ and let $N$ be the $k \times n$ matrix with $i$-th row $s_{i}$. Let $t$ be any row matrix in $P$ but not in $Q$. Then there exists a $k$-th order function $f$ defined only on the rows of $N^{T}$ such that $f\left(N^{T}\right)=t^{T}$. We wish to show that any predicate in $\mathscr{P}$ commutes with $f$. By way of contradiction suppose that the $m$-th order predicate $P_{1} \in \mathscr{P}$ does not commute with $f$ and that every predicate obtained from $P_{1}$ by identification of variables does commute with $f$. Then there exists a $j \times m$ matrix $N_{1} \subset P_{1}$ such that $f\left(N_{1}^{T}\right)=t_{1}^{T}$ and $t_{1}$ is not contained in $P_{1}$.

Since every identification of variables in $P$ leads to a predicate which commutes with $f$ we must have that each pair $r_{i}, f\left(r_{i}\right) i=$ $1, \cdots, m$ where $r_{i}$ is the $i$-th row of $N_{1}^{T}$ and $f\left(r_{i}\right)$ is the corresponding element of $t_{1}^{r}$, is distinct from any other pair $r_{j}, f\left(r_{j}\right)$. Thus each pair is the same as a row element pair taken from $N^{T}$ and $t^{T}$. We can find a $k \times n$ matrix $N_{2} \subset A^{n-m} P_{1}$ and row matrix $t_{2}$ such that the last $m$ rows of $N_{2}^{T}$ and elements of $t_{2}^{T}$ are equal to $r_{i}, f\left(r_{i}\right)$. Also the first $n-m$ pairs can be chosen so that there is a one to one correspondence between pairs taken from $N^{T}, t^{T}$ and pairs taken from $N_{2}^{T}, t_{2}^{T}$. By permuting the variables of $A^{n-m} P_{1}$ we can arrive at a predicate $P_{3}$ which contains $N$ and does not contain $t$. Since $P_{3}$ is in $\mathscr{P}$ we get that $P$ is not the least $n$-th order predicate which contains $Q$. Thus we have a contradiction and $f$ must commute with every predicate of $\mathscr{P}$. Thus $\mathscr{P}=\mathscr{P}^{* *}$.

Now we consider systems of predicates which are closed under the existential quantifier. Let $\mathscr{L}$ be a closed system of functions and assume that for any $f\left(x_{1}, \cdots, x_{n}\right) \in \mathscr{L}$ with restricted domain of definition, there exists a $g\left(x_{1}, \cdots, x_{n}\right) \in \mathscr{L}$ which is defined on all of $A^{n}$ and equals $f$ where $f$ is defined. Then it can be verified that $\mathscr{L}^{*}$ is closed under the existential quantifier.

THEOREM 3. If $\mathscr{P}$ is a closed system of predicates which is 
closed under the existential quantifier then every function in $\mathscr{P}^{*}$ can be extended to a function in $\mathscr{P}^{*}$ which is defined for all values of the arguments.

We assume that the elements of $A$ are the integers from 1 to $n$. Let $f\left(x_{1}, \cdots, x_{m}\right) \in \mathscr{P}^{*}$ be defined on the sequences $s_{1}, s_{2}, \cdots, s_{k}$ and let $s$ be any other sequence in $A^{m}$. We define the $n$ functions $f_{i}$ such that $f_{i}\left(s_{j}\right)=f\left(s_{j}\right)$ and $f_{i}(s)=i$ for $i=1, \cdots, n$ and show that for some $i, f_{i}$ is in $\mathscr{P}^{*}$. By way of contradiction suppose that for each $f_{i}$ there exists a $P_{i} \supset N_{i}$ where $P_{i} \in \mathscr{P}$ and $N_{i}$ is a matrix such that $f_{i}\left(N_{i}^{T}\right)^{T}$ is not in $P_{i}$. We can assume that each $N_{i}$ has $s^{T}$ in the first column and every other column is an $s_{i}^{T}$, if $N_{i}$ has more than one occurence of $s^{T}$ then by identifying variables in $P_{i}$ we can arrive at a new $P_{i}$ which has only one occurence of $s^{T}$ in the corresponding $N_{i}$. Also after permuting the variables of $P_{i}$ we can assume that $s^{T}$ occurs as the first column of $N_{i}$. Let

$$
P_{1}\left(x, x_{1}, \cdots, x_{p}\right), P_{2}\left(x, y_{1}, \cdots, y_{q}\right), \cdots, P_{n}\left(x, z_{1}, \cdots, z_{r}\right)
$$

be the predicates which satisfy these conditions, since $\mathscr{P}$ is closed the predicate $P\left(x, x_{1}, \cdots, x_{p}, y_{1}, \cdots, y_{q}, \cdots, z_{1}, \cdots, z_{r}\right)$ equivalent to the conjunction of the $P_{i}$ is in $\mathscr{P}$. Also $P$ contains a matrix $N$ derived from the $N_{i}$ with first column $s^{T}$ and each remaining column equal to an $s_{i}^{T}$. Now $E P$ contains the matrix $N_{0}$ which is $N$ with its first column deleted. Since $E P$ is in $\mathscr{P}$ we have that $f\left(N^{T}\right)^{T}$ is in $E P$. Thus $P$ contains a sequence $i, f\left(N^{T}\right)^{T}$ for some $i$. But this contradicts the assumption that $f_{i}\left(N_{i}^{T}\right)^{T}$ is not in $P_{i}$. Thus $f$ can be extended to a function defined for all values of the variables.

Now we consider single valued functions which are defined for all values of their arguments. If $\mathscr{G}$ is a system of predicates we redefine $\mathscr{O}^{*}$ as the set of single valued functions defined for all values of the arguments which commute with $\mathscr{P}$. Also we assume that $\mathscr{P}$ is closed, contains $e\left(x_{1}, x_{2}\right) \Leftrightarrow\left(x_{1}=x_{2}\right)$ and is closed under the existential quantifier. We will give necessary and sufficient conditions on $\mathscr{S}^{*}$ in order that $\mathscr{P}$ be closed under disjunction and negation.

First we define the predicates $D\left(x_{1}, x_{2}, x_{3}, x_{4}\right) \Leftrightarrow\left(x_{1}=x_{2}\right) \vee\left(x_{3}=x_{4}\right)$ and $Q_{n}\left(x_{1}, \cdots, x_{n}\right)$ which holds in case $x_{i} \neq x_{j}$ for all $1 \leqq i<j \leqq n$. We have the following equivalences for a closed system $\mathscr{P}$.

(1) $\mathscr{P}^{*}$ consists of essentially first order functions if and only if $D \in \mathscr{P}$.

(2) When $\mathscr{P}$ is defined on a set $A$ with $n$ elements then $\mathscr{P}^{*}$ consists of essentially first order permutations if and only if $D, Q_{n} \in \mathscr{T}$.

We only prove that if $D \in \mathscr{P}$ then $\mathscr{P}^{*}$ consists of essentially first order functions. Let $g\left(x_{1}, \cdots, x_{n}\right)$ be a function in $\mathscr{P}^{*}$ which 
depends essentially on the variables $x_{1}$ and $x_{2}$. Then there exist sequences $\left(a_{1}, a_{2}, \cdots, a_{n}\right)=s_{1},\left(a_{0}, a_{2}, \cdots, a_{n}\right)=s_{2},\left(b_{1}, b_{2}, \cdots, b_{n}\right)=s_{3}$ and $\left(b_{1}, b_{0}, b_{3}, \cdots, b_{n}\right)=s_{4}$ such that $g\left(s_{1}\right) \neq g\left(s_{2}\right)$ and $g\left(s_{3}\right) \neq g\left(s_{4}\right)$. We construct the $4 \times n$ matrix $M$ with $i$-th row $s_{i}$. Then $M^{T} \subset D$ but $g(M)^{T}$ is not in $D$ so $g$ cannot be in $\mathscr{P}^{*}$. The other implications also follow easily. From these equivalences we get:

THEOREM 4. $\mathscr{P}$ is closed under disjunction if and only if $\mathscr{P}^{*}$ consists of essentially first order functions.

TheOREM 5. $\mathscr{P}$ is closed under negation if and only if $\mathscr{P}^{*}$ consists of first order permutations.

4. Comments and applications. First we consider the case where $A$ is an infinite set. Craig $\mathrm{R}$. Platt has found in this case that we need to add the following condition to the definition of closure of a set of functions or predicates. A set of functions $\mathscr{L}$ is locally closed if, for any $n$-th order function $g$ and for every finite $H \subset A^{n+1}$ there exists an $f \in \mathscr{L}$ such that $g \cap H=f \cap H$, then $g \in \mathscr{L}$. A similar definition is given for sets of predicates. Then it follows, if $\mathscr{L}$ and $\mathscr{P}$ are any sets of functions and predicates, that $\mathscr{L}^{*}$ and $\mathscr{P}^{*}$ are locally closed sets and Theorems 1 and 2 hold when $\mathscr{L}$ and $\mathscr{P}$ are locally closed. Also a theorem has been found in the infinite case which specializes to Theorem 3.

Theorems 1 and 2 can be summarized in the following way. Let $\mathscr{P}$ and $\mathscr{P}$ be the sets of all functions and predicates on a set and let $C$ be a binary relation which holds between elements in $\mathscr{L}$ and $\mathscr{P}$ if and only if they commute. Then $C$ is a difunctional relation $\left[1\right.$, p. 193] that is $C C^{*} C=C$. Here $C^{*}$ is the converse relation to $C$. Then $C C^{*}$ and $C^{*} C$ are congruence relations on $\mathscr{L}$ and $\mathscr{P}$ and $C$ establishes a one to one correspondence between the congruence classes. Alternately we may say that there exists a set $S$ and mappings $\phi: \mathscr{L} \rightarrow S$ and $\pi: \mathscr{P} \rightarrow S$ such that two elements $f \in \mathscr{L}$ and $P \in \mathscr{P}$ commute if and only if $\phi(f)=\pi(P)$.

In [2] Post has given a classification of two valued systems of functions. This gives a classification of two valued systems of predicates containing equality and closed under the existential quantifier. Finding these systems can be simplified using theorems of this paper.

The author wishes to thank the referee for his suggestions. 


\section{REFERENCES}

1. Oystein Ore, Theory of graphs, Amer. Math. Soc. Coll. Pub. 38, Amer. Math. Soc., Providence, R.I., 1962.

2. Emil Post, The two-valued iterative systems of mathematical logic, Ann. of Math. Stud. 5, Princeton University Press, Princeton, 1941.

Received September 28, 1967.

UNIVERSITY OF ILLINOIS 


\section{PACIFIC JOURNAL OF MATHEMATICS}

EDITORS

H. ROYDEN

Stanford University

Stanford, California

R. R. Phelps

University of Washington

Seattle, Washington 98105
J. DugundJI

Department of Mathematics

University of Southern California

Los Angeles, California 90007

\section{RICHARD ARENS}

University of California

Los Angeles, California 90024

\section{ASSOCIATE EDITORS}
E. F. BeCKENBACH
B. H. NeUmanN
F. WOLF
K. YOSIDA

\section{SUPPORTING INSTITUTIONS}

UNIVERSITY OF BRITISH COLUMBIA CALIFORNIA INSTITUTE OF TECHNOLOGY UNIVERSITY OF CALIFORNIA MONTANA STATE UNIVERSITY UNIVERSITY OF NEVADA NEW MEXICO STATE UNIVERSITY OREGON STATE UNIVERSITY UNIVERSITY OF OREGON OSAKA UNIVERSITY UNIVERSITY OF SOUTHERN CALIFORNIA
STANFORD UNIVERSITY UNIVERSITY OF TOKYO UNIVERSITY OF UTAH WASHINGTON STATE UNIVERSITY UNIVERSITY OF WASHINGTON

AMERICAN MATHEMATICAL SOCIETY CHEVRON RESEARCH CORPORATION TRW SYSTEMS NAVAL WEAPONS CENTER 


\section{Pacific Journal of Mathematics \\ Vol. 27, No. $1 \quad$ January, 1968}

Willard Ellis Baxter, On rings with proper involution ............... 1

Donald John Charles Bures, Tensor products of $W^{*}$-algebras........... 13

James Calvert, Integral inequalities involving second order derivatives . . . . 39

Edward Dewey Davis, Further remarks on ideals of the principal class.... 49

Le Baron O. Ferguson, Uniform approximation by polynomials with integral

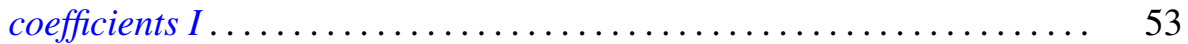

Francis James Flanigan, Algebraic geography: Varieties of structure

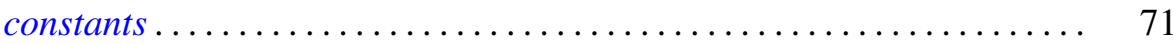

Denis Ragan Floyd, On QF -1 algebras ..................... 81

David Scott Geiger, Closed systems of functions and predicates ......... 95

Delma Joseph Hebert, Jr. and Howard E. Lacey, On supports of regular Borel measures ................................... 101

Martin Edward Price, On the variation of the Bernstein polynomials of a function of unbounded variation ........................ 119

Louise Arakelian Raphael, On a characterization of infinite complex matrices mapping the space of analytic sequences into itself........ 123

Louis Jackson Ratliff, Jr., A characterization of analytically unramified

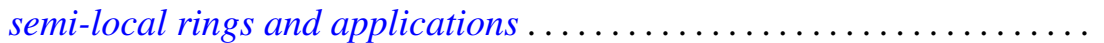

S. A. E. Sherif, A Tauberian relation between the Borel and the Lototsky transforms of series ................................ 145

Robert C. Sine, Geometric theory of a single Markov operator .......... 155

Armond E. Spencer, Maximal nonnormal chains in finite groups......... 167

Li Pi Su, Algebraic properties of certain rings of continuous functions .... 175

G. P. Szegô, A theorem of Rolle's type in $E^{n}$ for functions of the class $C^{1} \ldots 193$

Giovanni Viglino, A co-topological application to minimal spaces ........ 197

B. R. Wenner, Dimension on boundaries of $\varepsilon$-spheres ............... 201 